Moroccan J. of Pure and Appl. Anal. (MJPAA)

Volume 6(1), 2020, Pages 42-52

ISSN: Online 2351-8227 - Print 2605-6364

DOI 10.2478/mjpaa-2020-0004

\title{
Leray-Schauder's solution for a nonlocal problem in a fractional Orlicz-Sobolev space
}

\author{
Athmane BOUMAZOURH ${ }^{1}$, MoHAMmed SRATI ${ }^{2}$
}

\begin{abstract}
Aвstract. Via Leray-Schauder's nonlinear alternative, we obtain the existence of a weak solution for a nonlocal problem driven by an operator of elliptic type in a fractional Orlicz-Sobolev space, with homogeneous Dirichlet boundary conditions.
\end{abstract}

Mathematics Subject Classification (2010). MSC 35J20, 35J60, 35R11, 58E05.

Key words and phrases. Fractional $a$-laplacian, Fractional Orlicz-Sobolev spaces, Leray-Schauder's alternative theorem.

\section{Introduction}

In this paper, we aim to investigate the existence of solution for a class of quasilinear elliptic problem driven by a nonlocal integro-differential operator with homogeneous Dirichlet boundary data. In other words, we study the following problem

$$
\left(P_{a}\right)\left\{\begin{aligned}
(-\Delta)_{a}^{s} u=f(x, u) & \text { in } \quad \Omega, \\
u=0 & \text { in } \quad \mathbb{R}^{N} \backslash \Omega,
\end{aligned}\right.
$$

Received date 15 December 2019 - Accepted: 02 March 2020.

(C) This article is published with open access by Sidi Mohamed Ben Abdallah University.

The Author(s): ${ }^{1,2}$ Department of Mathematics, Sidi Mohamed Ben Abdellah University, Fez, P.O. Box 1796, Atlas 30000 Fez, Morocco.

1 e-mail:athmane.boumazourh@gmail.com

2 e-mail: mohammed.srati@usmba.ac.ma (Corresponding Author). 
where $\Omega$ is a bounded open subset of $\mathbb{R}^{N}, N \geqslant 1$, with Lipschitz boundary $\partial \Omega, f: \Omega \times \mathbb{R} \longrightarrow$ $\mathbb{R}$ is a Carathéodory function satisfying some growth conditions, and $(-\Delta)_{a}^{S}$ is the nonlocal fractional $a$-Laplacian operator of elliptic type which defined as

$$
(-\Delta)_{a}^{s} u(x)=2 \lim _{\varepsilon \searrow 0} \int_{\mathbb{R}^{N} \backslash B_{\varepsilon}(x)} a\left(\frac{|u(x)-u(y)|}{|x-y|^{s}}\right) \frac{u(x)-u(y)}{|x-y|^{s}} \frac{d y}{|x-y|^{N+s}},
$$

for all $x \in \mathbb{R}^{N}$, where $a: \mathbb{R} \longrightarrow \mathbb{R}$ which will be specified later.

Recently, a great attention has been given to the study of nonlocal fractional operators of elliptic type (see, for instance $[4,6,5,7])$. This type of operators arises in many different applications, such as, continuum mechanics, phase transition phenomena, population dynamics, minimal surfaces and game theory, as they are the typical outcome of stochastically stabilization of Levy processes, see for example $[13,18,17])$.

The study of quasilinear elliptic equations which involves homogeneous operators is based on the theory of Sobolev spaces $W^{1, p}(\Omega)$ and their corresponding fractional Sobolov spaces $W^{s, p}(\Omega)$ in order to find weak solutions. However, in the case of non-homogeneous differential operators, when we try to slot some conditions (growth conditions for example), problems have no correspondence in the classical Lebesgue and Sobolev spaces. This leads to use a more general class of functional spaces which called Orlicz-Sobolev spaces. These spaces consists of functions that have weak derivatives and satisfy certain integrability conditions. Since $\left(P_{a}\right)$ is a problem which involves the fractional a-Laplacian operator, the most appropriate functional framework to deal with it is the fractional Orlicz -Sobolev space.

When $a(t)=t^{p-2}$, the problem $\left(P_{a}\right)$ is reduced to the fractional $p$-Laplacian problem

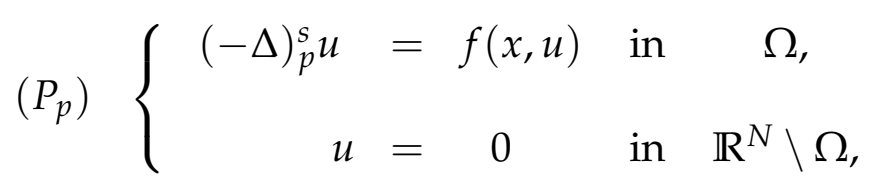

where $(-\Delta)_{p}^{S}$ is the fractional $p$-Laplacian operator which, up to normalization, defined as

$$
(-\Delta)_{p}^{s} u(x)=2 \lim _{\varepsilon \searrow 0} \int_{\mathbb{R}^{N} \backslash B_{\varepsilon}(x)} \frac{|u(x)-u(y)|^{p-2}(u(x)-u(y))}{|x-y|^{N+s p}} d y .
$$

One typical feature of problem $\left(P_{p}\right)$ is the nonlocality, in the sense that the value of $(-\Delta)_{p}^{S} u(x)$ at any point $x \in \Omega$ depends not only on the value of $u$ in a neighborhood of $x$, but on the value of $\mathrm{u}$ on the entire of $\mathbb{R}^{N}$. In recent years, the problem $\left(P_{p}\right)$ has been studied in many papers, we refer to $[2,16,21]$, in which the authors have used different methods to get the existence of solutions for $\left(P_{p}\right)$. For example in [24], by using Schauders fixed point theorem, Xiang et al studied the existence of solutions the problem $\left(P_{p}\right)$.

For the problems involving fractional $p$-Laplace operator, we refer the reader to the works $[8,9,10,11]$. they use different methods to establish the existence of solutions.

This paper is divided into four sections. In the second section, we recall some properties of fractional Orlicz Sobolev spaces. In the third section, applying several important properties of fractional $a$-Laplacian operator, we obtain the existence of weak solutions of problem $\left(P_{a}\right)$. 


\section{Some preliminaries results}

In this section, we briefly review the definitions and list some basic properties of the fractional Orlicz-Sobolev space. We refer the reader to $[1,3,12,14,23,19]$ for further reference and for some of the proofs of the results in this section.

Let $\Omega$ be an open subset of $\mathbb{R}^{N}, N \geqslant 1$. We assume that $a: \mathbb{R} \longrightarrow \mathbb{R}$ in $\left(P_{a}\right)$ is such that : $\varphi: \mathbb{R} \longrightarrow \mathbb{R}$ defined by :

$$
\varphi(t)=\left\{\begin{array}{ccc}
a(|t|) t & \text { for } & t \neq 0, \\
0 & \text { for } t=0,
\end{array}\right.
$$

is increasing homeomorphism from $\mathbb{R}$ onto itself. Let

$$
\Phi(t)=\int_{0}^{t} \varphi(\tau) d \tau
$$

Then, $\Phi$, is $N$-function, see [1], i.e. $\Phi: \mathbb{R}^{+} \longrightarrow \mathbb{R}^{+}$is continuous, convex, increasing function, with $\frac{\Phi(t)}{t} \rightarrow 0$ as $t \rightarrow 0$ and $\frac{\Phi(t)}{t} \rightarrow \infty$ as $t \rightarrow \infty$.

For the function $\Phi$ introduced above we define the Orlicz space :

$$
L_{\Phi}(\Omega)=\left\{u: \Omega \longrightarrow \mathbb{R} \text { mesurable } \int_{\Omega} \Phi(\lambda|u(x)|) d x<\infty \text { for some } \lambda>0\right\} .
$$

The space $L_{\Phi}(\Omega)$ is a Banach space endowed with the Luxemburg norm

$$
\|u\|_{\Phi}=\inf \left\{\lambda>0: \int_{\Omega} \Phi\left(\frac{|u(x)|}{\lambda}\right) d x \leqslant 1\right\} .
$$

The conjugate $N$-function of $\Phi$ is defined by $\bar{\Phi}(t)=\int_{0}^{t} \bar{\varphi}(\tau) d \tau$, where $\bar{\varphi}: \mathbb{R} \longrightarrow \mathbb{R}$ is given by $\bar{\varphi}(t)=\sup \{s: \varphi(s) \leqslant t\}$. Furthermore, it is possible to prove a Hölder type inequality, that is

$$
\left|\int_{\Omega} u v d x\right| \leqslant 2|| u\left\|_{\Phi}|| v\right\|_{\Phi} \quad \forall u \in L_{\Phi}(\Omega) \text { and } v \in L_{\bar{\Phi}}(\Omega) .
$$

Throughout this paper, we assume that

$$
1<\varphi^{-}:=\inf _{t \geqslant 0} \frac{t \varphi(t)}{\Phi(t)} \leqslant \varphi^{+}:=\sup _{t \geqslant 0} \frac{t \varphi(t)}{\Phi(t)}<+\infty .
$$

The above relation implies that $\Phi \in \Delta_{2}$ i.e. $\Phi$ satisfies the global $\Delta_{2}$-condition (see [22]) :

$$
\Phi(2 t) \leqslant K \Phi(t) \text { for all } t \geqslant 0,
$$

where $K$ is a positive constant.

Furthermore, we assume that $\Phi$ satisfies the following condition

$$
\text { the function }[0, \infty) \ni t \mapsto \Phi(\sqrt{t}) \text { is convex. }
$$

The above relation assures that $L_{\Phi}(\Omega)$ is an uniformly convex space (see [22]). 
Definition 2.1. Let $A, B$ be two N-function. $A$ is stronger (resp essentially stronger) than $B$, $A \succ B(\operatorname{resp} A \succ \succ B)$ in symbols, if

$$
B(x) \leqslant A(a x), \quad x \geqslant x_{0} \geqslant 0,
$$

for some (resp for each) $a>0$ and $x_{0}$ (depending on $a$ ).

Remark 1. (see. [1, Section 8.5]). $A \succ \succ B$ is equivalent to the condition

$$
\lim _{x \rightarrow \infty} \frac{B(\lambda x)}{A(x)}=0,
$$

for all $\lambda>0$.

Now, we defined the fractional Orlicz-Sobolev space $W^{s} L_{\Phi}(\Omega)$ as follows

$$
W^{s} L_{\Phi}(\Omega)=\left\{u \in L_{\Phi}(\Omega): \int_{\Omega} \int_{\Omega} \Phi\left(\frac{\lambda|u(x)-u(y)|}{|x-y|^{s}}\right) \frac{d x d y}{|x-y|^{N}}<\infty \text { for some } \lambda>0\right\} .
$$

This space is equipped with the norm,

$$
\|u\|_{s, \Phi}=\|u\|_{\Phi}+[u]_{s, \Phi},
$$

where $[\cdot]_{s, \Phi}$ is the Gagliardo seminorm, defined by

$$
[u]_{s, \Phi}=\inf \left\{\lambda>0: \int_{\Omega} \int_{\Omega} \Phi\left(\frac{|u(x)-u(y)|}{\lambda|x-y|^{s}}\right) \frac{d x d y}{|x-y|^{N}} \leqslant 1\right\} .
$$

To deal with the problem under consideration, we choose

$$
W_{0}^{s} L_{\Phi}(\Omega)=\left\{u \in W^{s} L_{\Phi}\left(\mathbb{R}^{N}\right): u=0 \text { a.e. } \mathbb{R}^{N} \backslash \Omega\right\} .
$$

which can be equivalently renormed by setting $\|\|=.[.]_{s, \Phi}$. By [3], $W_{0}^{s} L_{\Phi}(\Omega)$ is Banach space, also separable (resp. reflexive) space if and only if $\Phi \in \Delta_{2}$ (resp. $\Phi \in \Delta_{2}$ and $\bar{\Phi} \in \Delta_{2}$ ). Furthermore if $\Phi \in \Delta_{2}$ and $\Phi(\sqrt{t})$ is convex, then the space $W^{s} L_{\Phi}(\Omega)$ is uniformly convex.

Lemma 2. (see. [12]). Let $\Phi$ be an $N$-function which satisfies the global $\Delta_{2}$-condition. Then we have,

$$
\bar{\Phi}(\varphi(t)) \leqslant c \Phi(t) \text { for all } t \geqslant 0
$$

where $c>0$.

Proposition 3. (cf. [3]) Assume condition (2.2) is satisfied. Then the following relations holds true,

$$
\begin{aligned}
& {[u]_{s, \Phi}^{\varphi^{-}} \leqslant \phi(u) \leqslant[u]_{s, \Phi}^{\varphi^{+}}, \forall u \in W^{s} L_{\Phi}(\Omega) \text { with }[u]_{s, \Phi}>1,} \\
& {[u]_{s, \Phi}^{\varphi^{+}} \leqslant \phi(u) \leqslant[u]_{s, \Phi}^{\varphi^{-}}, \forall u \in W^{s} L_{\Phi}(\Omega) \text { with }[u]_{s, \Phi}<1,}
\end{aligned}
$$

where $\phi(u)=\int_{\Omega} \int_{\Omega} \Phi\left(\frac{|u(x)-u(y)|}{|x-y|^{s}}\right) \frac{d x d y}{|x-y|^{N}}$. 
In this paper, we assume the following conditions :

$$
\int_{0}^{1} \frac{\Phi^{-1}(\tau)}{\tau^{\frac{N+s}{N}}} d \tau<\infty
$$

and

$$
\int_{1}^{\infty} \frac{\Phi^{-1}(\tau)}{\tau^{\frac{N+s}{N}}} d \tau=\infty
$$

We define the inverse Sobolev conjugate $\mathrm{N}$-function of $\Phi$ as follows,

$$
\Phi_{*}^{-1}(t)=\int_{0}^{t} \frac{\Phi^{-1}(\tau)}{\tau^{\frac{N+s}{N}}} d \tau .
$$

Theorem 4. (cf. [3]) Let $\Omega$ be a bounded open subset of $\mathbb{R}^{N}$ with $C^{0,1}$-regularity and bounded boundary. If (2.4), (2.5) and (2.2) hold, then

$$
W^{s} L_{\Phi}(\Omega) \hookrightarrow L_{\Phi_{*}}(\Omega) .
$$

Theorem 5. (cf. [3]) Let $\Omega$ be a bounded open subset of $\mathbb{R}^{N}$ and $C^{0,1}$-regularity with bounded boundary. If (2.4), (2.5) and (2.2) hold, then

is compact for all $B \prec \prec \Phi_{*}$.

$$
W^{s} L_{\Phi}(\Omega) \hookrightarrow L_{B}(\Omega),
$$

Theorem 6. (cf. [3]) Let $\Omega$ be a bounded open subset of $\mathbb{R}^{N}$. Then,

$$
C_{0}^{2}(\Omega) \subset W^{s} L_{\Phi}(\Omega) .
$$

Finally, the proof of our main result is based on the following fixed point theorem:

Theorem 7. (Schauder fixed point [15]). Let $T$ be a continuous and compact mapping from a Banach space $X$ into itself, such that the set

$$
\{x \in X: x=\lambda T x \text { for some } 0 \leq \lambda \leq 1\}
$$

is bounded. Then $T$ has a fixed point.

\section{Mains Results}

In this section we prove the existence of solutions to problem $\left(P_{a}\right)$. Our approach is based on some properties of the operator $(-\Delta)_{a}^{s} u($.$) and on the nonlinear Leray Schauder alternative.$

Let $g$ an odd increasing homeomorphism function from $\mathbb{R}$ onto $\mathbb{R}$. By setting

$$
G(s)=\int_{0}^{s} g(t) d t, \quad \bar{G}(s)=\int_{0}^{s} \bar{g}(t) d t,
$$

where $\bar{g}(t)=\sup \{s: g(s) \leqslant t\}$, we obtain complementary N-functions which define corresponding Orlicz spaces $L_{G}$ and $L_{\bar{G}}$.

Then, we suppose that $f: \Omega \times \mathbb{R} \rightarrow \mathbb{R}$ is a Carathéodory function satisfying: 
$\left(f_{1}\right)$

$$
\left\{\begin{array}{r}
\text { There exists nonnegative constant } c_{1} \text { and such that } \\
|f(x, t)| \leqslant b(x)+c_{2} g(|t|) \text { for all } t \in \mathbb{R} \text { and a.e. } x \in \Omega,
\end{array}\right.
$$

where $b \in L_{\bar{G}}(\Omega)$. We will also assume that

$$
\begin{gathered}
1<q^{-}=\inf _{s>0} \frac{\operatorname{tg}(t)}{G(s)} \leqslant q^{+}=\sup _{s>0} \frac{\operatorname{tg}(t)}{G(s)}<+\infty . \\
\lim _{t \rightarrow \infty} \frac{G(k t)}{\Phi_{*}(t)}=0 \text { for all } k>0 .
\end{gathered}
$$

Remark 8. Condition $\left(f_{3}\right)$ implies that $G \prec \prec \Phi_{*}$. So by Theorem 5 , the following embedding :

$$
W_{0}^{s} L_{\Phi}(\Omega) \hookrightarrow L_{G}(\Omega)
$$

is compact, that is,

$$
\|u\|_{G} \leqslant c_{0}\|u\| \text { for all } u \in W_{0}^{s} L_{\Phi}(\Omega)
$$

where $c_{0}$ is a positive constant.

Definition 3.1. We say that $u \in W_{0}^{s} L_{\Phi}(\Omega)$ is a weak solution of problem $\left(P_{a}\right)$ if

$$
\int_{\Omega \times \Omega} a\left(\frac{|u(x)-u(y)|}{|x-y|^{s}}\right) \frac{u(x)-u(y)}{|x-y|^{s}} \frac{v(x)-v(y)}{|x-y|^{s}} \frac{d x d y}{|x-y|^{N}}=\int_{\Omega} f(x, u) v d x,
$$

for all $v \in W_{0}^{s} L_{\Phi}(\Omega)$.

Theorem 9. Assume that $f$ satisfies $\left(f_{1}\right)-\left(f_{3}\right)$ and we assume that $\Phi$ satisfies (2.2)- (2.5). Then, if $\varphi^{-}>2$ the problem $\left(P_{a}\right)$ has at least one weak solution $u \in W_{0}^{s} L_{\Phi}(\Omega)$.

First, we discus the fractional a-Laplace operator

$$
\mathcal{T} u:=(-\Delta)_{a}^{s} u(x)=2 p . v \int_{\mathbb{R}^{N}} a\left(\frac{|u(x)-u(y)|}{|x-y|^{s}}\right) \frac{u(x)-u(y)}{|x-y|^{s}} \frac{d y}{|x-y|^{N+s}} .
$$

To simplify the notation, we set

$$
h(u):=\frac{u(x)-u(y)}{|x-y|^{s}},
$$

and the dual space of $\left(W_{0}^{s} L_{\Phi}(\Omega),\|\|.\right)$ is denoted by $\left(\left(W_{0}^{s} L_{\Phi}(\Omega)\right)^{*},\|.\|_{*}\right)$.

Lemma 10.

1) $\mathcal{T}: W_{0}^{s} L_{\Phi}(\Omega) \longrightarrow\left(W_{0}^{s} L_{\Phi}(\Omega)\right)^{*}$ is continuous, bounded and strictly monotone operator.

2) $\mathcal{T}$ is a mapping of type $\left(S_{+}\right)$, that is, for a sequence $\left\{u_{n}\right\}$ that converges weakly to $u$ in $W_{0}^{s} L_{\Phi}(\Omega)$ and

$$
\limsup _{n \rightarrow \infty}\left\langle\mathcal{T} u_{n}, u_{n}-u\right\rangle \leqslant 0 \text {, }
$$

we have $u_{n} \longrightarrow$ u in $W_{0}^{s} L_{\Phi}(\Omega)$.

3) $\mathcal{T}: W_{0}^{s} L_{\Phi}(\Omega) \longrightarrow\left(W_{0}^{s} L_{\Phi}(\Omega)\right)^{*}$ is a homomorphism. 
Proof. 1) Consider the following functional :

$$
J(u)=\int_{\Omega \times \Omega} \Phi\left(\frac{|u(x)-u(y)|}{|x-y|^{s}}\right) \frac{d x d y}{|x-y|^{N}} .
$$

We know that (see [3]), $J \in C^{1}\left(W_{0}^{s} L_{\Phi}(\Omega), \mathbb{R}\right)$, and the fractional $a$-Laplace operator is the derivative operator of $J$ in the weak sense. We denote $\mathcal{T}=J^{\prime}: W_{0}^{s} L_{\Phi}(\Omega) \longrightarrow\left(W_{0}^{s} L_{\Phi}(\Omega)\right)^{*}$, then

$$
\langle\mathcal{T} u, v\rangle=\int_{\Omega \times \Omega} a\left(\frac{|u(x)-u(y)|}{|x-y|^{s}}\right) \frac{u(x)-u(y)}{|x-y|^{s}} \frac{v(x)-v(y)}{|x-y|^{s}} \frac{d x d y}{|x-y|^{N}} .
$$

Obviously that $\mathcal{T}$ is continuous and bounded. Let $u, v \in W_{0}^{s} L_{\Phi}(\Omega)$, with $u \neq v$ and let $\lambda, \mu \in[0,1]$ with $\lambda+\mu=1$. Since $a(|t|) t$ is increasing and :

$$
\langle\mathcal{T} u-\mathcal{T} v, u-v\rangle=\int_{\Omega} \int_{\Omega}(a(|h(u)|) h(u)-a(|h(v)|) h(v))(h(u)-h(v)) \frac{d x d y}{|x-y|^{N}}>0,
$$

So, $\mathcal{T}: W_{0}^{s} L_{\Phi}(\Omega) \longrightarrow\left(W_{0}^{s} L_{\Phi}(\Omega)\right)^{*}$ is strictly monotone.

2) Since $u_{n}$ converges weakly to $u$ in $W_{0}^{S} L_{\Phi}(\Omega)$, then $\left\{\left\|u_{n}\right\|\right\}$ is a bounded sequence of real numbers, this and Proposition 3, imply that $\left\{J\left(u_{n}\right)\right\}$ is bounded, then we have for a subsequence noted also $u_{n}$,

$$
J\left(u_{n}\right) \longrightarrow c \text {. }
$$

Since $J$ is weak lower semi continuous, we get

$$
J(u) \leqslant \liminf _{n \rightarrow \infty} J\left(u_{n}\right)=c .
$$

On the other hand, by the convexity of $J$, we have

$$
J(u) \geqslant J\left(u_{n}\right)+\left\langle J^{\prime}\left(u_{n}\right), u_{n}-u\right\rangle .
$$

Taking into account (3.2), we obtain

$$
J(u)=c .
$$

Since $\left\{\frac{u_{n}+u}{2}\right\}$ converges weakly to $u$ in $W_{0}^{s} L_{\Phi}(\Omega)$, so since $J$ is sequentially weakly lower semicontinuous :

$$
c=J(u) \leqslant \liminf _{n \rightarrow \infty} J\left(\frac{u_{n}+u}{2}\right) .
$$

Assume by contradiction that $\left\{u_{n}\right\}$ does not converge to $u$ in $W_{0}^{s} L_{\Phi}(\Omega)$. Hence, there exists a subsequence of $\left\{u_{n}\right\}$, still denoted by $\left\{u_{n}\right\}$ and there exists $\varepsilon_{0}>0$ such that

$$
\left\|\frac{u_{n}-u}{2}\right\| \geqslant \frac{\varepsilon_{0}}{2},
$$

by Proposition 3, we have

$$
J\left(\frac{u_{n}-u}{2}\right) \geqslant \max \left\{\varepsilon_{0}^{\varphi^{-}}, \varepsilon_{0}^{\varphi^{+}}\right\}
$$


On the other hand, by the conditions (2.2) and (2.3), we apply Lemma 2.1 in [20] to obtain

$$
\frac{1}{2} J\left(u_{n}\right)+\frac{1}{2} J(u)-J\left(\frac{u_{n}+u}{2}\right) \geqslant J\left(\frac{u_{n}-u}{2}\right) \geqslant \max \left\{\varepsilon_{0}^{\varphi^{-}}, \varepsilon_{0}^{\varphi^{+}}\right\} .
$$

It follows from (3.5) that

$$
J(u)-\max \left\{\varepsilon_{0}^{\varphi^{-}}, \varepsilon_{0}^{\varphi^{+}}\right\} \geqslant \limsup _{n \rightarrow \infty} J\left(\frac{u_{n}+u}{2}\right) .
$$

Thus, from (3.4) and (3.6) we obtain a contradiction, and hence $\left\{u_{n}\right\}$ converges strongly to $u$.

3) First, we assume that the operator $\mathcal{T}: W_{0}^{s} L_{\Phi}(\Omega) \longrightarrow\left(W_{0}^{s} L_{\Phi}(\Omega)\right)^{*}$ is invertible. By the Minty-Browder theorem (see [25]), it suffices to prove that $\mathcal{T}$ is strictly monotone, hemicontinuous and coercive in the sense of monotone operators.

From 1) the operator, $\mathcal{T}: W_{0}^{s} L_{\Phi}(\Omega) \longrightarrow\left(W_{0}^{s} L_{\Phi}(\Omega)\right)^{*}$ is strictly monotone. Let $u \in W_{0}^{S} L_{\Phi}(\Omega)$, with $\|u\|>1$, By (2.2) and Proposition 3, we have $\varphi^{-} \Phi(t) \leqslant \varphi(t) t$ for all $t>0$ and

$$
\begin{aligned}
\frac{\langle\mathcal{T} u, u\rangle}{\|u\|} & \geqslant \frac{\varphi^{-} J(u)}{\|u\|} \\
& \geqslant\left.\varphi^{-}\|u\|\right|^{\varphi^{-}-1}
\end{aligned}
$$

Thus

$$
\lim _{\|u\| \rightarrow \infty} \frac{\langle\mathcal{T} u, u\rangle}{\|u\|}=\infty
$$

so, $\mathcal{T}$ is coercive.

Now, from 1), $\mathcal{T}$ is hemicontinuous. Thus, in view of the Minty-Browder theorem, there exists $\mathcal{T}^{-1}:\left(W_{0}^{s} L_{\Phi}(\Omega)\right)^{*} \longrightarrow W_{0}^{s} L_{\Phi}(\Omega)$ and it is bounded.

Let us prove that $\mathcal{T}^{-1}$ is continuous by showing that its is sequentially continuous. Let $\left\{u_{n}\right\} \subset$ $\left(W_{0}^{s} L_{\Phi}(\Omega)\right)^{*}$ be a sequence strongly converging to $u \in\left(W_{0}^{s} L_{\Phi}(\Omega)\right)^{*}$ and let $v_{n}=\mathcal{T}^{-1}\left(u_{n}\right)$ and $v=\mathcal{T}^{-1}(u)$. Then, $\left\{v_{n}\right\}$ bounded in $W_{0}^{s} L_{\Phi}(\Omega)$, so, it converges weakly to a certain $v_{0} \in W_{0}^{s} L_{\Phi}(\Omega)$. Since $u_{n}$ converges strongly to $u$, we have

$$
\lim _{n \rightarrow \infty}\left\langle\mathcal{T}\left(v_{n}\right), v_{n}-v_{0}\right\rangle=\lim _{n \rightarrow \infty}\left\langle u_{n}, v_{n}-v_{0}\right\rangle=0,
$$

that is,

$$
\int_{\Omega} \int_{\Omega} a\left(h\left(v_{n}\right)\right) h\left(v_{n}\right)\left(h\left(v_{n}\right)-h_{x, y}\left(v_{0}\right)\right) \frac{d x d y}{|x-y|^{N}}=0 .
$$

By applying 2), using (3.7) and the fact that $v_{n}$ converges weakly to $v_{0}$ in $W_{0}^{s} L_{\Phi}(\Omega)$, we conclude that $v_{n}$ converge strongly to $v_{0}$ in $W_{0}^{s} L_{\Phi}(\Omega)$. Thus, this complete the proof.

Proof of Theorem 9 From Remark 8, $W_{0}^{s} L_{\Phi}(\Omega)$ compactly embedded in $L_{G}(\Omega)$. Denote by $i$ the compact injection of $W_{0}^{s} L_{\Phi}(\Omega)$ in $L_{G}(\Omega)$ and $i^{*}: L_{\bar{G}}(\Omega) \longrightarrow\left(W_{0}^{s} L_{\Phi}(\Omega)\right)^{*}, i^{*} v=v o i$ for all $v \in L_{\bar{G}}(\Omega)$, its adjoint. Its follows from the assumption $\left(f_{1}\right)$ that the Nemytskii operator $N_{f}$ generated by $f,\left(N_{f} u\right)(x)=f(x, u(x))$ is well defined from $L_{G}(\Omega)$ into $L_{\bar{G}}(\Omega)$, continuous and bounded. In oder to prove that problem $\left(P_{a}\right)$ has a week solution $u \in W_{0}^{s} L_{\Phi}(\Omega)$, it's sufficient to prove that the equation

$$
\mathcal{T} u=\left(i^{*} N_{f} i\right) u
$$


has a solution in $W_{0}^{s} L_{\Phi}(\Omega)$. Indeed, if $u \in W_{0}^{s} L_{\Phi}(\Omega)$ is a solution of (3.8), then for all $v \in$ $W_{0}^{S} L_{\Phi}(\Omega)$, one has

which can be rewritten :

$$
\langle\mathcal{T} u, v\rangle=\left\langle\left(i^{*} N_{f} i\right) u, v\right\rangle=\left\langle N_{f}(i u), i v\right\rangle
$$

$$
\int_{\Omega \times \Omega} a\left(\frac{|u(x)-u(y)|}{|x-y|^{s}}\right) \frac{u(x)-u(y)}{|x-y|^{s}} \frac{v(x)-v(y)}{|x-y|^{s}} \frac{d x d y}{|x-y|^{N}}=\int_{\Omega} f(x, u) v d x
$$

so, $u \in W_{0}^{s} L_{\Phi}(\Omega)$ is a weak solution of problem $\left(P_{a}\right)$.

By Lemma (10), $\mathcal{T}$ is a homeomorphism from $W_{0}^{s} L_{\Phi}(\Omega)$ into $\left(W_{0}^{s} L_{\Phi}(\Omega)\right)^{*}$. The equation (3.8) is equivalent to

$$
u=\mathcal{T}^{-1}\left(i^{*} N_{f} i\right) u
$$

Therefore, proving that the problem $\left(P_{a}\right)$ has a weak solution in $W_{0}^{s} L_{\Phi}(\Omega)$ remains to show that the compact operator:

$$
\mathcal{L}:=\mathcal{T}^{-1}\left(i^{*} N_{f} i\right): W_{0}^{s} L_{\Phi}(\Omega) \longrightarrow\left(W_{0}^{s} L_{\Phi}(\Omega)\right)^{*},
$$

has a fixed point.

By Theorem 7, to prove that $\mathcal{L}$ admit a fixed point it sufficient to verifies the following property: There exists constant $R>0$ such that

$$
S=\left\{u \in W_{0}^{s} L_{\Phi}(\Omega): u=\lambda \mathcal{L} u \text { for some } \lambda \in[0,1]\right\} \subset B(0, R) .
$$

For $\lambda=0$, the only solution of equation $u=\lambda \mathcal{L} u$ is $u=0$.

For $\lambda \in(0,1]$, let $u \in W_{0}^{s} L_{\Phi}(\Omega)$ which satisfies $u=\lambda \mathcal{T}^{-1}\left[\left(i^{*} N_{f} i\right) u\right]$. Then, we have

$$
\langle\mathcal{T} u, u\rangle=\lambda\left\langle\left(i^{*} N_{f} i\right) u, u\right\rangle \text {. }
$$

If $\|u\|<1$, by $\left(f_{1}\right)$ and Proposition 3, we have $p^{-} \Phi(t) \leqslant \varphi(t) t$ for all $t>0$ and

$$
\begin{aligned}
p^{-}\|u\|^{p^{-}} & \leqslant p^{-} \int_{\Omega \times \Omega} \Phi\left(\frac{|u(x)-u(y)|}{|x-y|^{s}}\right) \frac{d x d y}{|x-y|^{N}} \\
& \leqslant\langle\mathcal{T} u, u\rangle \\
& \leqslant\left\langle\left(i^{*} N_{f} i\right) u, u\right\rangle \\
& \leqslant\left\|i^{*}\right\|_{*}\left\|N_{f}(i u)\right\|_{L_{\bar{G}}}|| u \|_{G} \\
& \leqslant c_{0}\left\|i^{*}\right\|_{*}\left\|N_{f}(i u)\right\|_{L_{\bar{G}}}\|u\| .
\end{aligned}
$$

In order to estimate $\left\|N_{f}(i u)\right\|_{L_{\bar{G}}}$, we deduce first from assumption $\left(f_{1}\right)$ that

$$
\left|\left(N_{f} v\right)(x)\right| \leqslant c_{1} g(|v|)+b(x)
$$

so, by Lemma 2 we have

$$
\left\|N_{f} v\right\|_{L_{\bar{G}}} \leqslant c_{2}\left(c_{1}\|v\|_{L_{G}}+\|b\|_{L_{\bar{G}}}\right) \text {, }
$$

where $c_{2}$ is a positive constant. By taking (3.11) for $v=i u, u \in W_{0}^{s} L_{\Phi}(\Omega)$, we have

$$
\left\|N_{f} v\right\|_{L_{\bar{G}}} \leqslant c_{2}\left(c_{1}\|i\|_{*}\|u\|+\|b\|_{L_{\bar{G}}}\right) \text {. }
$$

In particular, if $u \in W_{0}^{S} L_{\Phi}(\Omega)$, for some $\lambda \in(0,1]$, we conclude from (3.9) - (3.10) - (3.12) that 


$$
\begin{aligned}
p^{-}\|u\|^{p^{-}} & \leqslant c_{0} c_{2}\left\|i^{*}\right\|_{*}\left(c_{1}\|i\|\left\|_{*}\right\| u\|+\| b \|_{L_{\bar{G}}}\right)\|u\| . \\
& \leqslant c_{3}\|u\|^{2}+c_{4}\|u\|,
\end{aligned}
$$

where $c_{2}=c_{0} c_{1} c_{2}\left\|i^{*}\right\|_{*}\|i\|_{*}$, and $c_{3}=c_{0} c_{2}\left\|i^{*}\right\|_{*}\|b\|_{L_{\bar{G}}}$. Applying Young's inequality, we get

$$
c_{3}\|u\|^{2} \leqslant \frac{1}{4}\|u\|^{p^{-}}+4 \frac{2}{p^{-}-2} c_{3}^{\frac{p^{-}}{p^{-}-2}}
$$

and

$$
c_{4}\|u\| \leqslant \frac{1}{4}\|u\|^{p^{-}}+4^{\frac{1}{p^{-}-1}} c_{4}^{\frac{p^{-}}{p^{-}-1}} .
$$

Inserting these two inequalities into (3.13), one has

$$
\frac{1}{2}\|u\|^{p^{-}} \leqslant 4^{\frac{2}{p^{-}-2}} c_{3}^{\frac{p^{-}}{p^{-}-2}}+4^{\frac{1}{p^{-}-1}} c_{4}^{\frac{p^{-}}{p^{-}-1}}
$$

Set

$$
R_{1}=\left(2^{\frac{p^{-}+2}{p^{-}-2}} c_{3}^{\frac{p^{-}}{p^{-}-2}}+2^{\frac{p^{-}+1}{p^{-}-1}} c_{4}^{\frac{p^{-}}{p^{-}-1}}\right)^{\frac{1}{p^{-}}},
$$

then $\|u\| \leqslant R_{1}$. Similarly if $\|u\| \geqslant 1$ we have $\|u\| \leqslant R_{2}$ where

$$
R_{2}=\left(2^{\frac{p^{+}+2}{p^{-}-2}} c_{3}^{\frac{p^{+}}{p^{+}-2}}+2^{\frac{p^{+}+1}{p^{+}-1}} c_{4}^{\frac{p^{+}}{p^{+}-1}}\right)^{\frac{1}{p^{+}}}
$$

this implies that the set $S$ is bounded.

\section{References}

[1] R. A. Adams, Sobolev Spaces, Academic Press, New York, 1975.

[2] V. Ambrosion, Nontrivial solutions for a fractional $p$-Laplacian problem via Rabier Theorem. Journal of Complex Variables and Elliptic Equation, Volume 62, 2017, 838-847.

[3] E. Azroul, A. Benkirane, M.Srati, Existence of solutions for a nonlocal type problem in fractional Orlicz Sobolev spaces, Adv. Oper. Theory (2020) doi: 10.1007/s43036-020-00042-0.

[4] E. Azroul, A. Benkirane, M. Shimi, Eigenvalue problems involving the fractional $p(x)$-Laplacian operator. Adv. Oper. Theory 4 (2019), no. 2, 539-555. doi:10.15352/aot.1809-1420.

[5] E. Azroul, A. Benkirane, M. Shimi and M. Srati, On a class of fractional $p(x)$-Kirchhoff type problems. Applicable Analysis (2019) doi: 10.1080/00036811.2019.1603372.

[6] E. Azroul, A. Benkirane and M. Srati, Three solutions for Kirchhoff problem involving the nonlocal fractional pLaplacian. Adv. Oper. Theory (2019) doi: 10.15352/AOT.1901-1464.

[7] E. Azroul, A. Benkirane, A. Boumazourh and M. Srati, Three solutions for a nonlocal fractional p-Kirchhoff Type elliptic system. Applicable Analysis (2019) doi: 10.1080/00036811.2019.1670347.

[8] E. Azroul, A. Boumazourh and M. Srati, On a positive weak solutions for a class of weighted $(p(),. q()$.$) -Laplacian$ systems. Moroccan J. of Pure and Appl. Anal. (MJPAA) doi: 10.2478/mjpaa-2019-0010 (2020) 125-139

[9] E. Azroul, M. Shimi, Nonlocal eigenvalue problems with variable exponent, Moroccan J. of Pure and Appl. Anal, Volume 4(1), 2018, Pages 46-61, DOI 10.1515/mjpaa-2018-0006

[10] E. Azroul, A. Benkirane and M. Srati, Three solutions for a Schrödinger-Kirchhoff type equation involving nonlocal fractional integro-defferential operators J. Pseudo-Differ. Oper. Appl. (2020). https://doi.org/10.1007/s11868-02000331-5. 
[11] E. Azroul, A. Benkirane and M. Shimi, Existence and Multiplicity of solutions for fractional $p(x,$.$) -Kirchhoff$ type problems in $\mathbb{R}^{N}$, Applicable Analysis, (2019), DOI:10.1080/00036811.2019.1673373.

[12] J. F. Bonder and A. M. Salort, Fractional order Orlicz-Soblev spaces, Journal of Functional Analysis, 2019,

[13] Y. Chen, S. Levine, M. Rao, Variable exponent linear growth functionals in image processing, SIAM J. Appl. Math., 66 (2006), 1383-1406.

[14] F.Demengel ans G. Demengel Functional Spaces for the Theory of Elliptic Partial Differential Equations, Springer (2012).

[15] J. Dugundji, A. Granas; Fixed Point Theory. I. Monografie Matematyczne, vol. 61. PWN, Warsaw (1982)

[16] G. Franzina and G. Palatucci, Fractional p-eigenvalues, Riv. Math. Univ. Parma (N.S.) 5 (2014), no. 2, 373-386.

[17] T. C. Halsey, Electrorheological fluids, Science, 258 (1992), 761-766. https://doi.org/10.1016/j.jfa.2019.04.003.

[18] L. Diening, Theorical and numerical results for electrorheological fluids, Ph.D. thesis, University of Freiburg, Germany (2002).

[19] M. A. Krasnosel'skii and Ja. B. Rutickii, Convex functions and Orlicz spaces, Translated from the first Russian edition by Leo F. Boron, P. Noordhoff Ltd., Groningen, 1961. MR 0126722.

[20] J. Lamperti, On the isometries of certain function-spaces, Pacific J. Math. 8 (1958), 459-466.

[21] E. Lindgren and P. Lindqvist, Fractional eigenvalues, Calc. Var. 49 (2014) 795-826.

[22] M. Mihäilescu, V. Rädulescu, Neumann problems associated to nonhomogeneous differential operators in OrliczSoboliv spaces, Ann. Inst. Fourier 58 (6) (2008) 2087-2111.

[23] E. Di Nezza, G. Palatucci, and E. Valdinoci, Hitchhiker's guide to the fractional Sobolev spaces, Bull. Sci. Math. 136 (2012), no. 5, 521-573. MR 2944369.

[24] H. Qiu and M. Xiang; Existence of solutions for fractional p-Laplacian problems via Leray-Schauders nonlinear alternative; Boundary Value Problems (2016) DOI 10.1186/s13661-016-0593-8

[25] E. Zeidler, Nonlinear Functional Analysis and Applications, In Nonlinear monotone operators, Vol. II/B, Springer-Verlag, New York, 1990. 